\title{
An Anti-occlusion Video Target Tracking Method Based on Kalman Filter
}

\author{
Dang Kexin, Zhang Xiongfei, Chen Zhihong*, Yang Ziwen, Li Chen \\ School of Electrical and Electronic Engineering, Tianjin University of Technology, Tianjin, China \\ Email address: \\ chenzhihong_tjut@163.com (Chen Zhihong),386159737@qq.com (Dang Kexin) \\ ${ }^{*}$ Corresponding author
}

To cite this article:

Dang Kexin, Zhang Xiongfei, Chen Zhihong, Yang Ziwen, Li Chen. An Anti-occlusion Video Target Tracking Method Based on Kalman Filter. Journal of Electrical and Electronic Engineering. Vol. 7, No. 2, 2019, pp. 69-74. doi: 10.11648/j.jeee.20190702.17

Received: April 14, 2019; Accepted: May 23, 2019; Published: June 15, 2019

\begin{abstract}
The human visual system is the main component of the brain-based information processing system. It is the main way for humans to obtain external information. Therefore, the emerging science represented by computer vision came into being. Video-specific target tracking is a core issue in the field of computer vision research. It has been widely studied and concerned, and it has been widely used in many fields, such as video surveillance, intelligent navigation, medical diagnosis, augmented reality and virtual reality, etc. The specific moving target tracking algorithm and improved algorithm in the video are studied to some extent. Combining the meanshift algorithm with Kalman filtering can solve the occlusion problem of moving targets in complex scenes. This project intends to use Kalman filter and meanshift algorithm to detect and track the specified target in the video image sequence, and obtain the position, angle, scale, velocity, acceleration and dynamic trajectory of the target. Experiments show that this method has a good tracking effect on the target tracking in the video which is partially occluded during the motion.
\end{abstract}

Keywords: Computer Vision, Video Target Tracking, Kalman Filter, Signal Processing, Pattern Recognition

\section{Introduction}

\subsection{Research Background and Significance}

From the 20th century to the 21 st century, all kinds of information gradually expanded around us, and the extremely important image information of human beings flourished at an alarming rate [1]. With the continuous development of information technology, video surveillance systems will be increasingly needed in the territorial areas of life, industry and commerce, defense security, and military applications.

The research of computer vision originated in the 1960s, the early research is mainly aimed at two-dimensional images, through edge detection [2-4], target detection [5-6] and other pattern recognition methods to understand the image. In 1982, Marr [7] proposed a set of perfect computer vision theory to describe the visual process, which has become a very important research framework in the field of computer vision research. The study of computer vision has two meanings, first, in order to meet the needs of the application of artificial intelligence, which USES the computer realization of human visual system need these results above can be installed in all kinds of robot, the robot can have the ability to see. The second, the visual results of the calculation model, in return for our further understanding the human visual system [8] itself also has considerable reference value [9].

International attention is also attached to the research of computer vision, and conferences are held every year to update their existing knowledge. Each International Conference on Computer Vision (ICCV), European Computer Vision Conference (ECCV), and Computer Vision and Pattern Recognition Conference (CVPR) and other top-level computer vision conferences related to target detection and target tracking [10] papers will become the focus of the meeting [11].

Video target tracking [12] is an important research topic in the field of computer vision. Video target tracking research in real life, such as intelligent transportation, intelligent video surveillance, human-computer interaction, robot, face recognition, vehicle and pedestrian detection, medical navigation surgery, intelligent fitting room, etc. Basically including all aspects of food, clothing, housing and 
transportation, has a strong practical value. In the new era, the research of video target tracking has made a rapid development and presents many new characteristics. This is mainly due to the development of machine learning theory and technology and the construction of large-scale tracking data sets and evaluation platforms. The main task of video target tracking is to obtain the position and motion information of the objects of interest in the video sequence, which provides the basis for further semantic layer analysis, motion recognition and scene recognition. It is defined as: given the position frame of the target in the initial frame of the video sequence (usually a rectangular frame), the location frame of the target is automatically given in the subsequent video sequence, or a prompt is given when the target is out of view. In this paper, in order to make up for the inaccuracy of location caused by occlusion, the Kalman filter [13] is used to predict the optimal search window of the current frame, in which adaptive meanshift algorithm is used to determine the final position of the target in the current frame. It is proved by experiment that the combination of the above two can achieve good tracking effect.

It has five major computer vision techniques: image classification, object detection, target tracking, semantic segmentation and case segmentation. Target tracking plays an important role in computer vision. While video surveillance system motion target detection integrates image processing, pattern recognition, artificial intelligence, computer science and many other fields of advanced technology, has become an important field of computer vision research [14]. But we often encounter various problems, in order to improve the accuracy of target detection and tracking. The tracking of the target is affected by the distortion of the target itself, the interference of the complex background, various noises, occlusion, illumination and other factors. In the complex reality environment, the accuracy of the tracking algorithm can be improved pertinently. Robustness and real-time are the key points of the target tracking research [15]. Therefore, it is very important to analyze and describe the video image for how to accurately and effectively propose the moving target from the monitoring scene, and how to realize the real-time tracking, recognition and the understanding and description of the moving behavior of the target [16]. And the tracking systems must address two basic problems: motion and matching. Motion problem: predict the location of an image element being tracked in the next frame, that is, identify a limited search region in which the element is expected to be found with high probability. Matching problem: (also known as detection or location) identify the image element in the next frame within the designated search region [17].

\subsection{Status of Research}

In the field of intelligent monitoring, the research starts late and lags behind in domestic and foreign countries. Since 1986, the Chinese Academy of Sciences, Zhejiang University, Wuhan University and other universities and research institutes have made a lot of achievements in the detection and identification of sports pedestrians and the tracking of moving targets [18]. Some practical intelligent traffic monitoring systems are developed, which can be used in community, highway and so on.

There are many tracking algorithms in the field of visual tracking, which can meet the requirements of some application background, Target tracking algorithms can be roughly divided into feature-based [19], kernel-based [20], Bayesian [21] and detection-based [22] algorithms.

In 1975, Fukunaga et al first proposed the concept of meanshift in a paper [23] on the estimation of probability density gradient function. With the continuous development of image equipment, image tracking technology is becoming more and more perfect. In the image tracking technology, the image sensor can be used to obtain the continuous image sequence, and then the relevant information of the target motion, such as position, linear velocity, attitude Angle, etc., can be obtained through the corresponding algorithm. In more advanced image tracking systems, ground image detection equipment consisting of image radar or telescope and CCD (Charge Coupled Devices) is usually used to obtain image sequence, and some typical edge detection operators are used to detect the image boundary and determine the location of the target [24].

At present, the mainstream tracking algorithms mainly include Kalman filter tracking [25], particle filter tracking [26] and kernel tracking [27], The advantages and disadvantages of various methods are different. Meanshift algorithm [28-30] is the most commonly used kernel tracking method, because of its outstan ding real-time, robustness to interference and so on has been widely used. Different tracking methods have their own advantages, and the target tracking process requires high real-time performance of the algorithm, so this paper mainly studies the meanshift algorithm and its improved algorithm.

\subsection{The Content and Significance of the Research}

With the rapid development of society, security issues are becoming increasingly important. Today, camera-based surveillance equipment has been deployed in many important places, such as airports, various large and small shopping malls, school exchanges, banks and so on. These monitoring devices play an important role in preventing and solving many of the social security problems. However, traditional visual surveillance systems have many insurmountable deficiencies. For example, real-time monitoring of many important places requires the staff to continuously monitor the display equipment to prevent pedestrians or objects in the place from posing a potential threat to the safety of the surrounding environment. However, due to the inevitable physiological factors, it is unlikely that people will continue to stare at the display of the monitoring equipment continuously, which leaves an opportunity for many threats that may occur in an instant. Even if a person can continuously stare at the display of the monitoring device, it takes a lot of manpower and material resources.

Therefore, the traditional visual monitoring system is 
increasingly unable to meet the needs of people, and the intelligent monitoring system has emerged in this context. Intelligent monitoring is the process of target detection, target classification, target tracking, analysis and understanding of video data collected by cameras using computer vision technology. Based on this, the video surveillance system is controlled to make the video surveillance system look like a human. The intelligence can be used to monitor a scene and detect suspicious target activities or unexpected events. Intelligent visual surveillance has great application prospects in many important places, such as airports, various large and small shopping malls, schools, banks, etc. Therefore, moving target tracking is a vital part of intelligent monitoring research. The research of target tracking is the basis for realizing the application of intelligent visual monitoring in practice.

Video analysis technology based on moving target tracking is a hot topic in today's society. The meanshift algorithm is a commonly used excellent moving target tracking algorithm. This paper improves the deficiencies of the traditional meanshift algorithm. For example, the size of the target tracking frame cannot be changed, and the target is in poor tracking when it is moving fast or being occluded.

In view of the fact that the meanshift algorithm can not track the fast moving target and the target is being tracked, this paper combines the Kalman filter with the meanshift in the prediction function of the moving target, and each time the meanshift algorithm is executed. The Kalman filter is used to predict the position information of the target, and then the meanshift algorithm is used to further correct the target position, which enhances the judgment mechanism of the target position information, making the target tracking more effective.

\section{Improvement of Meanshift Algorithm}

Meanshift algorithm is a kind of kernel density estimation method. It does not need any prior knowledge and completely relies on the density function value of the sample points in the feature space. For a group of sampled data, the histogram method usually divides the range of data into several equal intervals, and the data is divided into several groups according to the interval. The ratio between the number of data in each group and the number of total parameters is the probability value of each unit. The kernel density estimation method is similar to the histogram method in principle, only one kernel function is added to smooth the data. The kernel function estimation method is used to estimate the density of data subject to any distribution.

The traditional meanshift algorithm is used for target tracking. The first step is to initialize the target tracking. It can get the external rectangle frame of the initial target to be tracked by the method of target detection, or it can be manually selected by the mouse. in this experiment, we use the mouse to select the target area manually. Then the histogram distribution of the search window weighted by the kernel function is calculated, and the histogram distribution of the corresponding window of the $\mathrm{N}$ frame is calculated by the same method. the search window moves in the direction of maximum density increase to get the true location of the target.

The meanshift algorithm tracking steps are as follows:

(1) Calculate probability density of target template $\left\{\mathrm{q}_{\mathrm{u}}\right\} \mathrm{u}=1 \ldots \mathrm{m}$, the estimated position of the target and the width of the nuclear window;

(2) Initialize the target position of the current frame with $\mathrm{y}_{0}$, initialize the target position of the current frame with $\mathrm{y}_{0}\left\{\mathrm{p}_{\mathrm{u}}\left(\mathrm{y}_{0}\right)\right\} \mathrm{u}=1 \ldots \mathrm{m}$;

(3) Calculate the weights of each point in the current window;

(4) Calculate the new position of the target.

But meanshift algorithm has great defects. Once the target is covered by a large area, or the background gray level changes greatly, the color information of the target model calculated by the traditional meanshift target tracking method cannot accurately reflect the gray level information of the target, which eventually leads to the tracking target being inaccurate or even missing In other words, When the moving target is too fast in the videos or the moving target is blocked, or the background color is similar to the target color, then the moving target will be lost. So we improved the meanshift algorithm by adding kalman filter. Kalman filter is used to predict the state vector of the current frame based on the observation vector of the previous frame so as to know the possible position of the target. Kalman filter is an optimal filter for the minimum mean square error estimation of the state sequence of a dynamic system. It can predict the center of the next frame area and correct the target area of the current frame. Its implementation principle is:

(1) Select a frame of video material as the background image, model the background image and calculate the difference between the next frame image and the background frame image to get the moving target

(2) In the initial search window, Kalman filter is used to predict the position of the moving target in the next frame

(3) The color probability distribution map is established with the backward projection method, and meanshift is nested with Kalman filter to calculate the optimal position of the moving target in the next frame

(4) Compared with the optimal value calculated by camshift, Kalman filter is updated. If there is occlusion of moving targets, the predicted value of Kalman filter is taken as the observed value, and the Kalman filter is updated at the same time

(5) Step 2-4 of the cycle: Keep track of the moving target

\section{Experimental Results and Analysis}

We tracked a white car coming from a distance. Firstly, the white car was selected with the red border. We found that as the car moved in video, the red border also moved with the white car. 


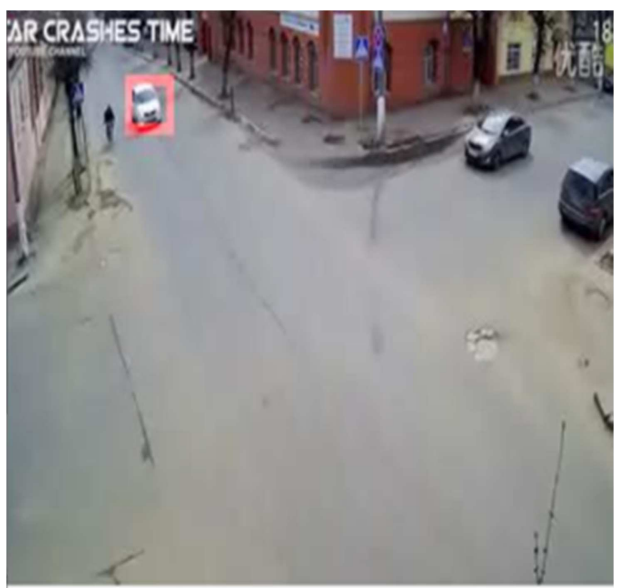

Figure 1. Preliminary target tracking diagram.

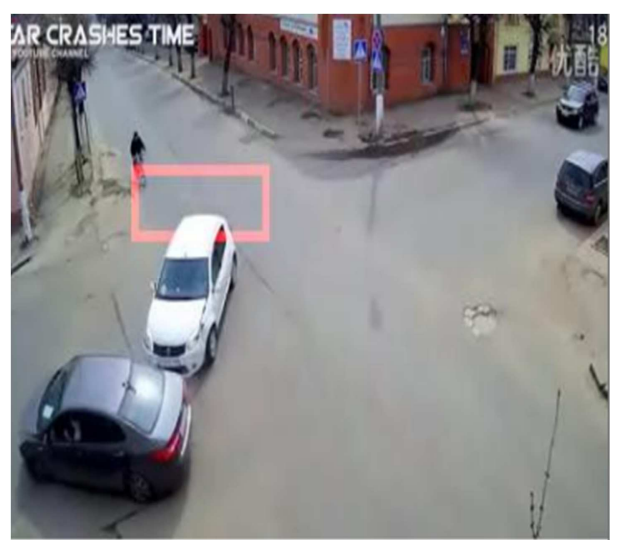

Figure 2. Preliminary target tracking diagram.

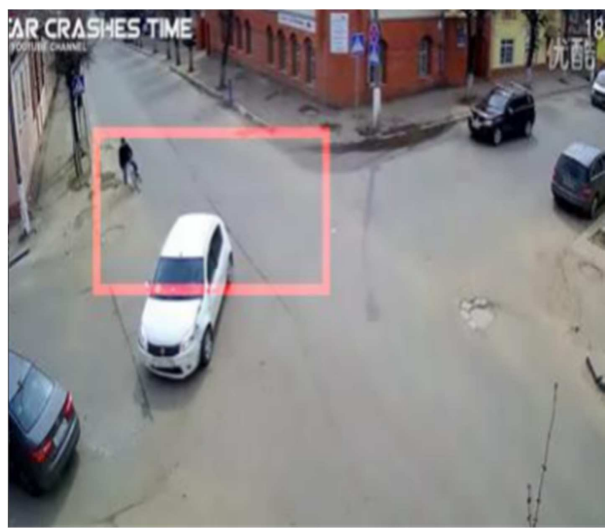

Figure 3. Preliminary target tracking diagram.

In Figure 2 and Figure 3. We found the effect of tracking wasn't optimistic enough and the captured target was slowly lost behind.

Based on the above motion analysis of the white car, we improved previous algorithm and integrated it with Kalman filter. We used the dynamic template to select the best region from Primary frames, and predicted the target area in the next frame by Kalman filter, then we got the new dynamic template according to the predicting result. Performance of the algorithm results can adaptively adjust the target area to continue tracking when the size of the target changes, the algorithm The results are shown in the following figures;

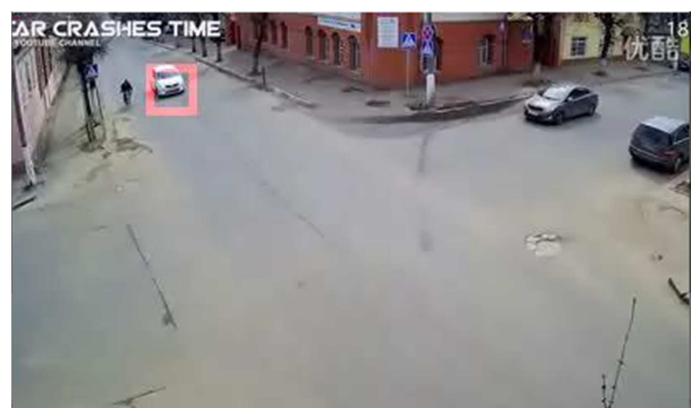

Figure 4. Improved target tracking diagram.

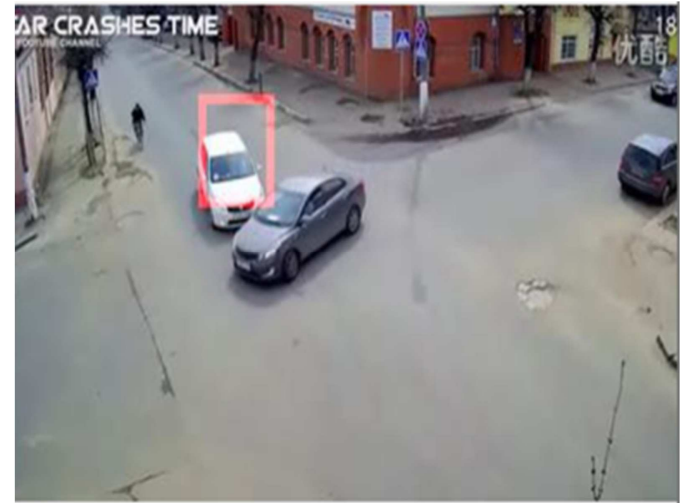

Figure 5. Improved target tracking diagram.

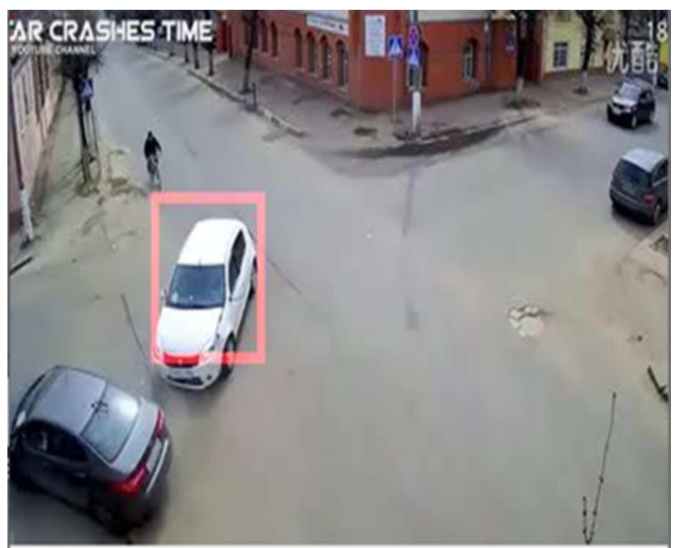

Figure 6. Improved target tracking diagram

From the figure, we can see that the tracking effect is very good, the target isn't lost and the target is not lost, the marker box changes correspondingly with the change of the target.

In the access of actual motion tracking, there will always be some noise due to environmental factors or other reasons, so that the tracking effect will become worse. People want to use the measurement results to estimate the motion as much as possible. In this way, the task can be divided into two stages: the first stage, the prediction stage, uses the information obtained in the past to further modify the position to obtain the next appearance of the target. In the second phase, the calibration phase, we take a measurement and adjust it to the expected value from the previous measurement.

When the target is covered, these are the tracking experiment contents: 


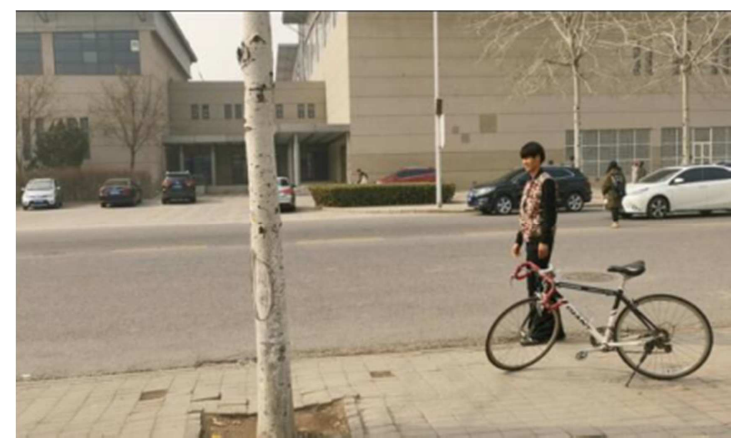

Figure 7. Original target state diagram.

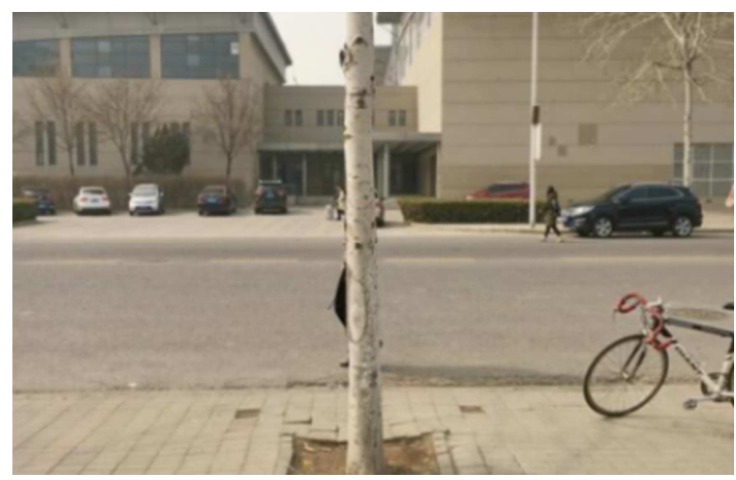

Figure 8. Original target state diagram.

From the pictures, there is an occlusion process in the processed video, and the target tracking process is carried out for video. The following figures show the results

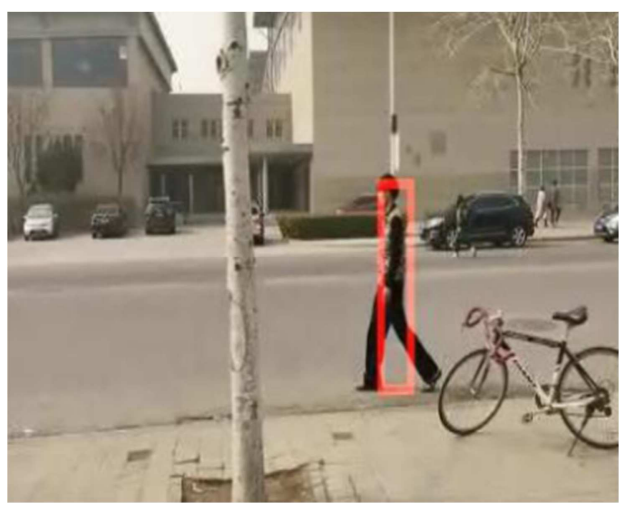

Figure 9. Improved target tracking diagram

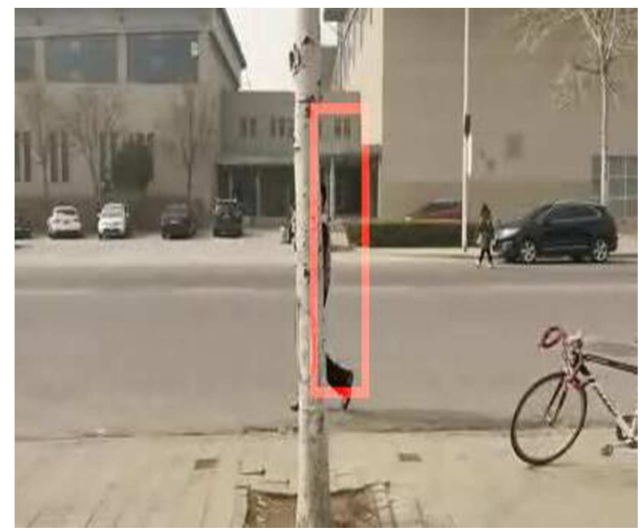

Figure 10. Improved target tracking diagram.

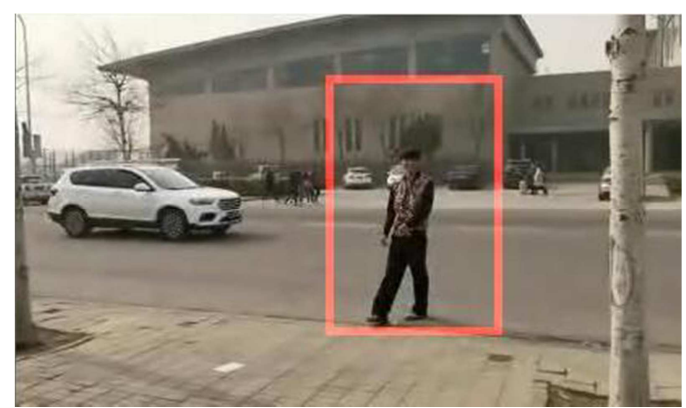

Figure 11. Improved target tracking diagram

From the experimental results, we know that the algorithm can also realize target tracking when the target has covered

\section{Summary and Discussion of the Experiment}

In this experiment, we deeply studied and implemented the traditional Mean Shift target tracking algorithm, and tested the tracking effect of the meanshift algorithm applied to targets of various motion types. The experimental results show that the traditional Mean Shift algorithm has better accuracy and robustness in the case of target partial occlusion and scale change. However, the algorithm tracks poorly or even fails when the target moves too fast or the target encounters severe occlusion. Aiming at the failure problem of meanshift algorithm in the case of target severe occlusion, an improved strategy combining meanshift and Kalman filter is proposed, which effectively solves the tracking problem of fast moving targets. Compared with the traditional meanshift algorithm, the tracking effect is very large improvement.

Specifically, we modeled the target motion and use the Kalman filter to predict the possible position of the target in the frame image based on the previous target position information. Then we can find the true location of the target near this location. In this way, we used the motion information of the previous frame to enrich the use of known information and enhance the tracking effect. As a result, it can be seen that the improved algorithm can achieve better tracking when the target encounters severe occlusion and reappears in the scene, which is greatly improved compared with the effect before the improvement. In the subsequent research, the video is The timeliness and robustness of specific target tracking raises higher requirements

\section{Acknowledgements}

Paper supported by the project of Tianjin university students' innovation and entrepreneurship (grant number 2017100600).

\section{References}

[1] Li anping. Research on video target tracking algorithm in complex environment [D]. Shanghai jiaotong university. 
[2] Guan hongrui, Ding hui. Research review of classical algorithms for image edge detection [J]. Journal of capital normal university: natural science edition, 2009 (S1): 66-69.

[3] Ma yan, zhang zhihui. Comparison of several edge detection operators [J]. Industrial and mining automation, 2004(1).

[4] Zhou xinming, Lan sai, $\mathrm{Xu}$ yan. Comparison of several edge detection algorithms in image processing [J]. Modern electric power, 2000, 17 (3): 65-69.

[5] Hyun, Eugin, and J. Lee. "Moving target range detection algorithm for FMCW radar." Radar Symposium IEEE, 2013.

[6] Wan ying, Han yi, Lu hanqing. Discussion on moving target detection algorithm $[\mathrm{J}]$. Computer simulation, 2006, 23 (10): 221-226.

[7] Marr, David. Vision: A Computational Investigation into the Human Representation and Processing of Visual Information [J]. Quarterly Review of Biology, 1982, 8.

[8] Liu yadong, Li Ming, Zhou zongtan, et al. Introduction of human visual system knowledge in the teaching of "computer vision" [J]. Journal of electrical and electronic education, 2010 32 (5): $10-11$

[9] Gao wen, Chen xilin. Computer vision: algorithm and system theory [M]. Tsinghua university press, 1999.

[10] Zhang Juan, Mao xiaobo, Chen tiejun. Research review of moving target tracking algorithm $[\mathrm{J}]$. Computer application research, 2009, 26 (12): 4407-4410.

[11] Guan hao, Xue xiangyang, An zhiyong. Application progress and prospect of deep learning in video target tracking [J]. Acta automatica sinica, 2016, 42 (6).

[12] CAI rongtai, Wu yuanhao, Wang mingjia, et al. Review of video target tracking algorithm [J]. Television technology, 2010 , 34 (12): 135-138.

[13] Song wenyao, Zhang ya. Kalman filter [M]. Science press, 1991.

[14] Li min. Research on moving target detection method in video monitoring system [D]. Northwest university, 2008.

[15] Gui zuheng. Research on video target tracking algorithm based on mean shift and particle filter [D]. Nanjing university of science and technology, 2009. Gu Hua, Su Guangda, du Cheng. Automatic location of key facial feature points [J]. Photoelectron. Laser

[16] Research on moving target detection and tracking algorithm in intelligent video monitoring system [D]. Jiangsu university, 2010.
[17] Guo yijiang. Detection and tracking of moving targets based on video [D]. East China normal university, 2009.

[18] Zhang jianghong. Analysis of the current situation of environmental monitoring at home and abroad $[\mathrm{J}]$. Science and fortune, 2015, 7 (33): 52-53.

[19] Qian lu. Research on target tracking algorithm based on feature fusion [D]. Zhejiang university of technology, 2014.

[20] Ning ji-feng, Jiang guang, Wu cheng-ke. Comparison and analysis of camshift and core-based target tracking algorithm $[\mathrm{J}]$. Computer engineering and application, 2009, 45 (28): $177-179$.

[21] Yin yili. Research on target tracking technology based on bayesian theory [D]. University of Chinese academy of sciences (xi 'an institute of optics and precision machinery, Chinese academy of sciences), 2016.

[22] Wu dongfei, Qi meibin. Moving target tracking algorithm based on detection [C]. measurement and control technology and instrumentation academic conference. 2012.

[23] Fukunaga K, Hostetler L D. The estimation of the gradient of a density function, with applications in pattern recognition [J]. IEEE Trans. inf. theory, 1975, 21 (1): 32-40.

[24] Chen yuanxiang. Research on video image moving target tracking technology [D]. Jiangsu university, 2010: 3-5.

[25] Fanhd, lixm. geometric interpretation of Kalman filtering algorithm [J]. Fire and command and control, 2002, 27 (4): 48-50. (in Chinese)

[26] Xiang ruxi, Li jianwei. Particle filter tracking algorithm based on multi-feature adaptive fusion [J]. Journal of computer aided design and graphics, 2012, 24 (1): 97-103. (in Chinese)

[27] Yang fan. An improved moving object tracking algorithm based on nuclear tracking $[\mathrm{J}]$. Computer and digital engineering, 2016, 44 (8): 1465-1467.

[28] Li X R, Wu F C, Hu Z Y. Convergence of a mean shift algorithm [J]. Journal of Software, 2005, 16 (3): 365-374.

[29] Wang tian, Liu weiting, Han guangliang, et al. Target tracking algorithm based on meanshift [J]. Liquid crystal display, 2012, 27 (3). 396-400.

[30] Tian xin. Research on meanshift algorithm based target tracking [D]. Xi 'an university of science and technology, 2010 . 